\title{
De novo formation of a large cavernoma associated with a congenital torcular dural arteriovenous fistula: case report
}

\author{
Waleed Brinjikji, MD, ${ }^{1}$ Kelly D. Flemming, MD, ${ }^{2}$ and Giuseppe Lanzino, MD² \\ Departments of ${ }^{1}$ Radiology, ${ }^{2}$ Neurology, and ${ }^{3}$ Neurosurgery, Mayo Clinic, Rochester, Minnesota
}

The authors report a case of a developmentally normal child with a congenital complex torcular dural arteriovenous fistula (DAVF) who later, in his teenage years, developed several vermian cavernomas within a large cerebellar developmental venous anomaly (DVA). The patient had initially presented with an abnormally large head circumference but no neurological deficits. He underwent several partial embolization procedures in an attempt to decrease the blood supply of the fistula over the course of 8 years. Nine years following initial presentation, he presented with a fourth ventricular hemorrhage, due to development of a new vermian cavernoma adjacent to a previously known vermian DVA and suffered subsequent mild left-sided hemiataxia from which he later recovered. CT angiographic images demonstrated that the vermian DVA drained into the left transverse sinus, which also drained the torcular arteriovenous fistula. A routine follow-up MRI examination 10 years following initial presentation demonstrated interval development of several large cavernomas in the cerebellum, all within the DVA. The patient had no new symptoms at that time and was neurologically intact. This case report highlights the de novo development of multiple cavernous malformations potentially secondary to DAVF-induced venous congestion in a preexisting DVA.

https://thejns.org/doi/abs/10.3171/2016.12.PEDS16600

KEY WORDS dural arteriovenous fistula; pediatrics; cavernous malformation; vascular disorders

$\mathrm{V}$ ENOus congestion of developmental venous anomalies (DVAs) associated with cerebral arteriovenous shunts can result in a variety of manifestations, including venous infarction, hemorrhage, or even de novo cavernoma formation. ${ }^{1-8}$ Herein we describe a case of a young male who presented with a torcular dural arteriovenous fistula (DAVF) that was associated with a DVA that eventually developed into a cluster of de novo cavernous malformations (CMs).

\section{Case Report}

This 6-year-old boy initially was brought to an outside institution for evaluation after his mother noted that he had a larger than normal head circumference. A noncontrast CT scan was performed (Fig. 1), revealing a mark- edly enlarged torcula. At the time of the initial evaluation, the patient had no cognitive impairment, pulsatile tinnitus, heart failure, or localizing neurological symptoms. He underwent a cerebral angiogram, which demonstrated a complex torcular DAVF that was supplied by bilateral posterior meningeal arteries, middle meningeal arteries, occipital arteries, and arteries of the tentorium cerebelli arising off of the meningohypophyseal trunk (Fig. 2). The fistula had antegrade venous drainage; there was no retrograde cortical venous drainage, but there was evidence of mild venous hypertension, with a pseudophlebitic pattern seen in late venous phase angiograms. Multiple transarterial embolizations were performed elsewhere over the course of an 8-year period in an attempt to decrease the blood supply to the high-flow fistula.

Eight years following initial presentation, a follow-up 
MRI examination demonstrated persistence of the fistula. Incidentally noted was a DVA that drained the vermis and left cerebellum (Fig. 3A). In retrospect, the cerebellar DVA was faintly visualized in the late venous phase of the initial angiogram obtained when the patient was 6 years old. A CT angiogram obtained at a later date again demonstrated the vermian and left cerebellar DVA, which drained into the left transverse sinus, just distal to the torcula (Fig. 3B). At that time there was no CM associated with the DVA. Nine years following initial presentation, the patient presented with headache and was found to have a new hemorrhage in the fourth ventricle (Fig. 3C). An MRI study performed at that time showed a cluster of CMs that had developed in the cerebellar vermis within the DVA (Fig. 3D). The patient developed a mild left hemiataxia as a result of the hemorrhage but eventually made a near-complete recovery. A repeat cerebral angiogram demonstrated no significant change in the appearance of the torcular DAVF with no substantial change in its venous drainage patterns.

Ten years following initial presentation, the patient underwent a routine MRI follow-up, which demonstrated significant enlargement of the vermian CM and left cerebellar tonsil, all within the region of the patient's known DVA. Interestingly, the patient had reported no new neuro-

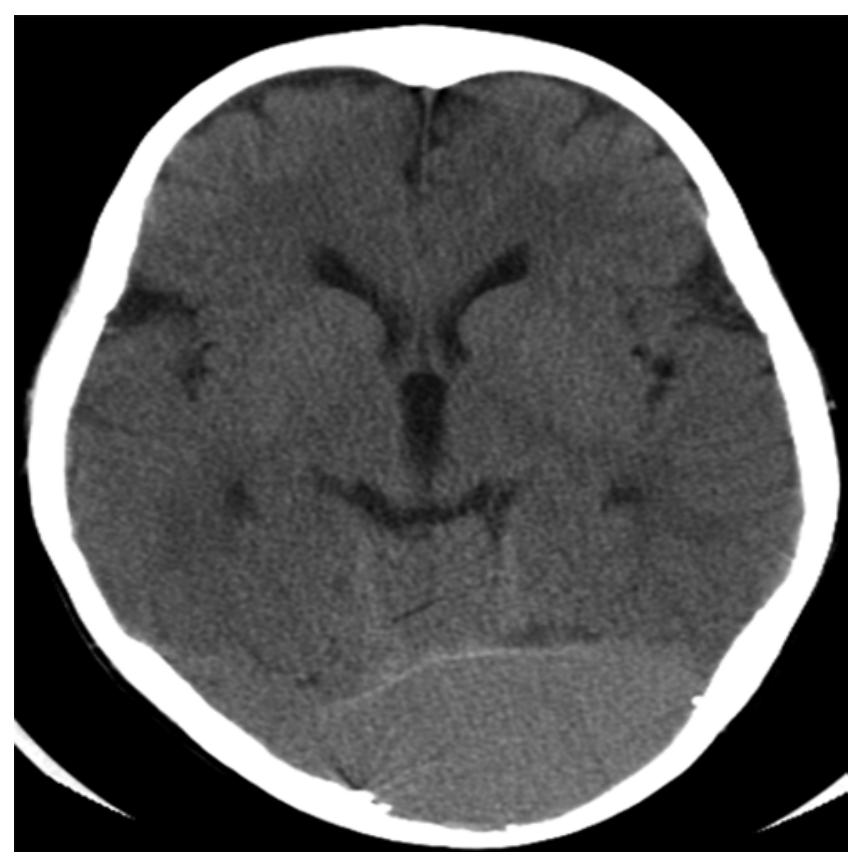

FIG. 1. Noncontrast axial CT image obtained at the time of presentation revealing a markedly enlarged torcula, the result of the patient's DAVF.
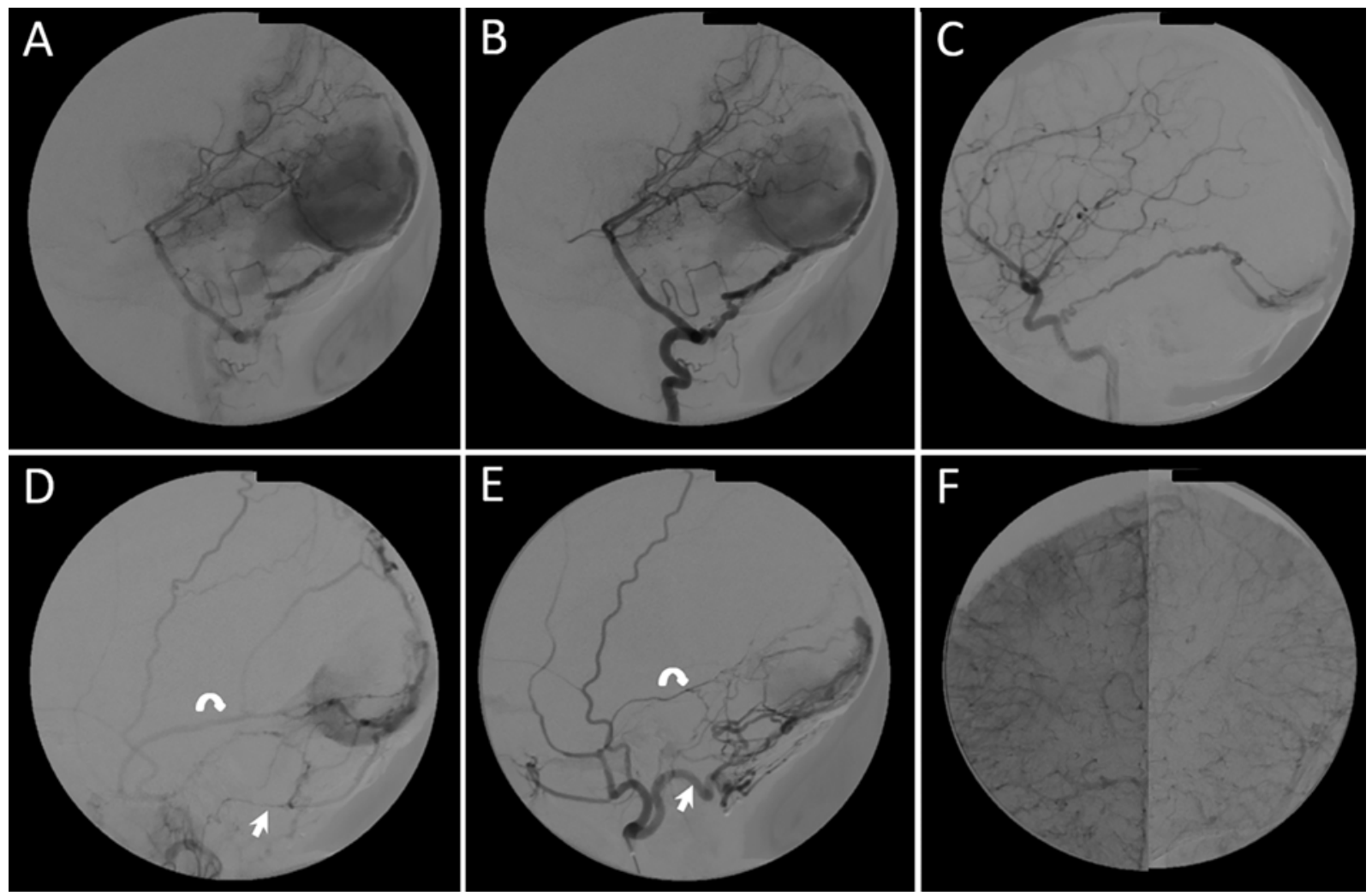

FIG. 2. Initial angiographic images. A: Left vertebral artery (VA) injection angiogram demonstrating marked hypertrophy of the posterior meningeal artery, which forms a direct fistula with the posterior aspect of the torcula. B: Right VA injection angiogram demonstrating a markedly hypertrophied right posterior meningeal artery, which also forms a direct arteriovenous fistula with the torcula. C: Right internal carotid artery (ICA) injection angiogram showing a markedly hypertrophied meningohypophyseal trunk and tentorial artery supplying the torcular DAVF. D: Right external carotid artery (ECA) injection angiogram showing supply to the DAVF arising from the posterior division of the middle meningeal artery (curved arrow) and the occipital artery (straight arrow). E: Left ECA injection angiogram showing supply to the DAVF arising from the posterior division of the middle meningeal artery (curved arrow) and the occipital artery (straight arrow). F: Late venous phase images of the bilateral ICA injections showing a pseudophlebitic pattern consistent with sequelae of chronic venous hypertension. 

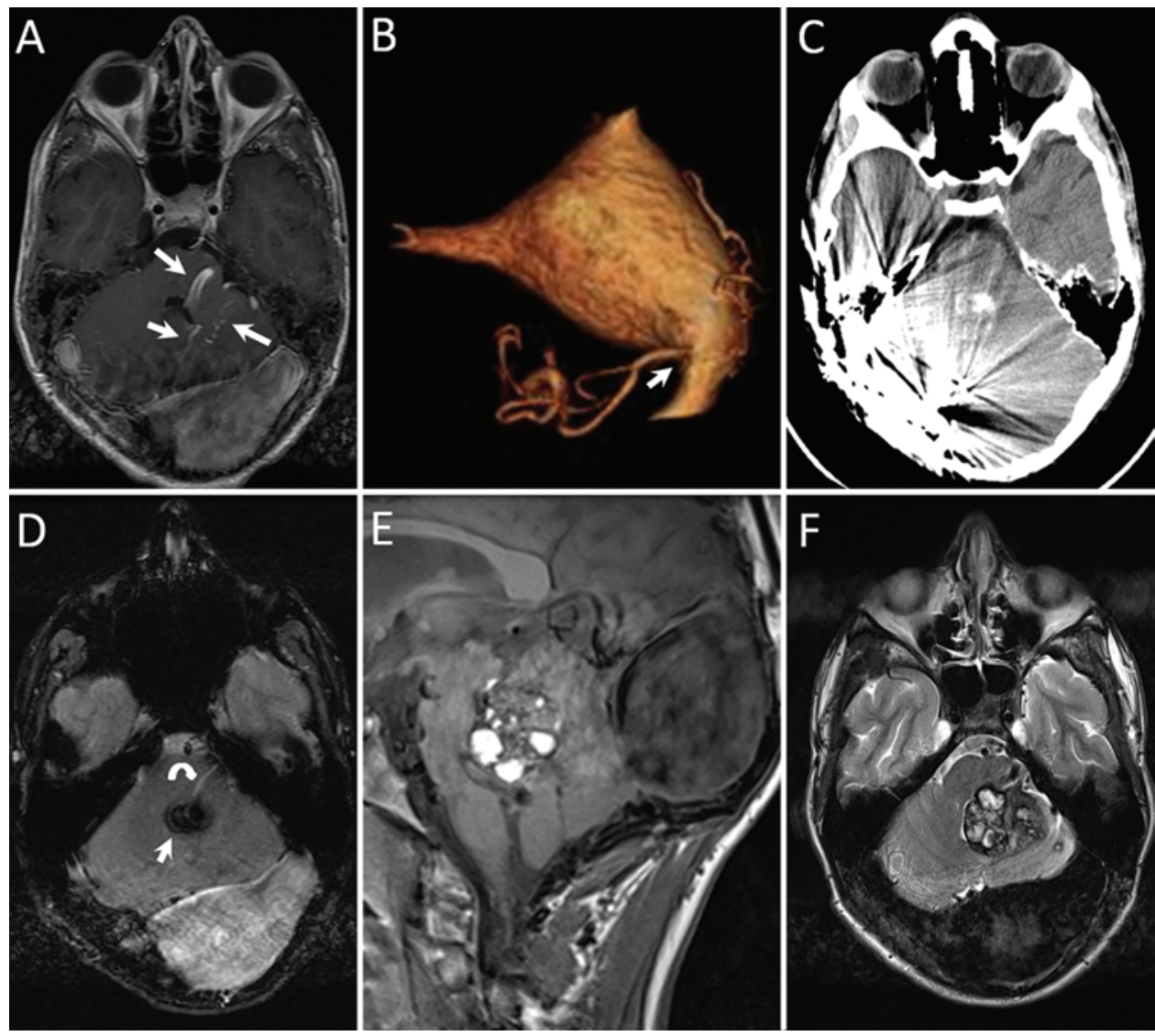

FIG. 3. DVA and cavernoma development. A: Axial contrast-enhanced T1-weighted MR angiogram showing a DVA of the vermis and left cerebellar hemisphere with multiple small venous radicles (arrows). B: 3D reconstruction of CT angiogram showing the DVA draining into the proximal left transverse sinus (arrow), sharing the same venous drainage as the torcula. C: Noncontrast axial CT image obtained 9 years following initial presentation showing a new hemorrhage in the fourth ventricle. D: Axial contrast-enhanced T1-weighted MR image obtained at the time of the hemorrhage showing 2 well-circumscribed spherical lesions in the vermis (arrow) with an associated draining vein, most consistent with a cavernoma. E and F: Sagittal T1-weighted (E) and axial T2-weighted (F) MR images obtained at the time of last follow-up (10 years following initial presentation), showing interval development of a large cluster of cavernomas in the vermis and left cerebellum. Figure is available in color online only.

logical symptoms, and neurological examination showed no significant abnormalities (Fig. 3E and F). Specifically, his gait (including tandem gait) and ocular movements were completely normal. Repeat cerebral angiography at this time again demonstrated no substantial change in the appearance of the DAVF and no change in its venous drainage pattern.

\section{Discussion}

We report on a patient with a DVA sharing a similar venous drainage pathway with a large torcular DAVF that eventually developed into a cluster of CMs, likely secondary to venous congestion due to the marked venous outflow from the DAVF. Both the DVA and the DAVF were likely congenital in nature and coexisted for over a decade prior to the formation of a cluster of CM.

Many prior studies and case reports have reported on de novo formation of CMs in the setting of DVAs. ${ }^{79}$ The presumed mechanism of a DVA resulting in the formation of a CM is elevated venous pressure in the territory of the DVA resulting in a cascade of physiological and signaling events leading to the formation of a CM. ${ }^{7}$ Prior studies have reported that CMs associated with DVAs often develop in the distal venous radicles of the DVA rather than within the large central DVA itself. ${ }^{1}$ This was seen in our patient, who had multiple CMs in the distal radicles of the DVA while the DVA remained patent.

DVA-associated de novo CM formation in the setting of a DAVF or other cerebral arteriovenous shunting lesion has been reported in prior case reports. ${ }^{2,4}$ In some cases the shunt drains directly into the DVA, ${ }^{4}$ while in others the DVA is adjacent to, and shares a common venous drainage pathway with, the arteriovenous shunt. ${ }^{2}$ Venous congestion within a DVA associated with an arteriovenous shunt can result in a variety of other neurological manifestations, including venous infarct and hemorrhage. ${ }^{5}$ One of the keys to managing DVAs associated with arteriovenous shunts is to maintain the patency of the DVA so as not to impair venous drainage of normal brain parenchyma. ${ }^{2,4,5}$ These cases, including ours, suggest that indeed venous hypertension may be causally related to the development of a CM in the presence of a DVA, rather than suggesting a purely casual association. This observation may shed some light into the mechanisms underlying the formation 
of a CM in association with a DVA, factors which so far have remained unknown and purely speculative.

One potential limitation of our case report is the fact that we do not have histopathological confirmation that the lesions within the cerebellar vermis were indeed CMs, rather than evolving hemorrhage. However, one would expect that hemorrhage would become smaller and less complex over time whereas in this case the hemorrhagic lesions within the vermis became larger and more complex and demonstrated the characteristic MRI findings of cavernomas, including a "popcorn" or "berry" appearance with a hemosiderin rim that bloomed on susceptibilityweighted images. In addition, acute hemorrhage would typically be associated with clinical symptoms. Lastly, whether the association between the DVA and the torcular AVF was truly the cause of the CM cannot be definitively established.

In conclusion, this case report highlights the de novo development of multiple CMs secondary to DAVF-induced venous congestion in a preexisting DVA.

\section{References}

1. Campeau NG, Lane JI: De novo development of a lesion with the appearance of a cavernous malformation adjacent to an existing developmental venous anomaly. AJNR Am J Neuroradiol 26:156-159, 2005

2. Chakravarthy H, Lin TK, Chen YL, Wu YM, Yeh CH, Wong HF: De novo formation of cerebral cavernous malformation adjacent to existing developmental venous anomaly - an effect of change in venous pressure associated with management of a complex dural arterio-venous fistula. Neuroradiol J 29:458-464, 2016

3. Fok KF, Holmin S, Alvarez H, Ozanne A, Krings T, Lasjaunias PL: Spontaneous intracerebral hemorrhage caused by an unusual association of developmental venous anomaly and arteriovenous malformation. Interv Neuroradiol 12:113121,2006
4. Geibprasert S, Krings T, Pereira V, Lasjaunias P: Infantile dural arteriovenous shunt draining into a developmental venous anomaly. Interv Neuroradiol 13:67-74, 2007

5. Komiyama M, Yamanaka K, Iwai Y, Yasui T: Venous angiomas with arteriovenous shunts: report of three cases and review of the literature. Neurosurgery 44:1328-1335, 1999

6. Kuncz A, Vörös E, Varadi P, Bodosi M: Venous cerebral infarction due to simultaneous occurrence of dural arteriovenous fistula and developmental venous anomaly. Acta Neurochir (Wien) 143:1183-1184, 2001

7. Pereira VM, Geibprasert S, Krings T, Aurboonyawat T, Ozanne A, Toulgoat F, et al: Pathomechanisms of symptomatic developmental venous anomalies. Stroke 39:3201-3215, 2008

8. Roh JE, Cha SH, Lee SY, Jeon MH, Cho BS, Kang MH, et al: Atypical developmental venous anomaly associated with single arteriovenous fistula and intracerebral hemorrhage: a case demonstrated by superselective angiography. Korean J Radiol 13:107-110, 2012

9. Zhang P, Liu L, Cao Y, Wang S, Zhao J: Cerebellar cavernous malformations with and without associated developmental venous anomalies. BMC Neurol 13:134, 2013

\section{Disclosures}

Dr. Lanzino reports a consultant relationship with Medtronic/ Covidien.

\section{Author Contributions}

Conception and design: all authors. Acquisition of data: all authors. Analysis and interpretation of data: all authors. Drafting the article: Brinjikji. Critically revising the article: all authors. Reviewed submitted version of manuscript: all authors. Approved the final version of the manuscript on behalf of all authors: Brinjikji.

\section{Correspondence}

Waleed Brinjikji, Department of Radiology, Mayo Clinic, 200 1st St. SW, Rochester, MN 55905. email: brinjikji.waleed@mayo.edu. 